\title{
Low-Dose Scanning Electron Diffraction Microscopy of Mechanochemically Nanostructured Pharmaceuticals
}

\author{
Duncan N. Johnstone ${ }^{1 *}$, Christopher S. Allen ${ }^{2,3}$, Mohsen Danaie ${ }^{3}$, Royston C.B. Copley ${ }^{4}$, Jeffrey Brum ${ }^{5}$, \\ Angus I. Kirkland ${ }^{2,3}$, Paul A. Midgley ${ }^{1}$ \\ ${ }^{1 .}$ Department of Materials Science \& Metallurgy, University of Cambridge, Cambridge, UK. \\ 2. Department of Materials, University of Oxford, Oxford, UK. \\ 3. Electron Physical Sciences Imaging Centre (ePSIC), Diamond Light Source, Harwell, UK. \\ 4. Physical Properties, CMC Analytical, GlaxoSmithKline Pharmaceuticals, Stevenage, UK. \\ 5. Physical Properties, CMC Analytical, GlaxoSmithKline Pharmaceuticals, Collegeville, USA. \\ * Corresponding author: dnj23@ cam.ac.uk
}

The solid-state structure of active pharmaceutical ingredients (APIs) is typically characterized in terms of polymorphism, co-crystallization, salt formation and solvate formation, which are known to have a significant effect on physical, chemical, mechanical and biopharmaceutical properties [1]. These structural features are studied in great detail, primarily using X-ray diffraction, optical spectroscopy, thermal analysis and nuclear magnetic resonance spectroscopy [1]. However, structural heterogeneity on the nanoscale (i.e. nanostructure) including crystal defects such as dislocations and stacking faults also effect properties but are challenging to study with these bulk techniques [1]. (Scanning) transmission electron microscopy ((S)TEM) is a natural choice for nanoscale characterization but, until recently, beam damage has been a major limiting factor in application to organic solids, including most APIs. Here, we report on the continued development of low-dose scanning electron diffraction (SED) microscopy, which has recently been shown to be a successful route to overcome these limitations [2, 3], and its application to reveal nanostructure in mechanochemically transformed pharmaceutical powders.

SED microscopy involves the acquisition of a two-dimensional electron diffraction pattern at every probe position as a nm-sized electron probe is scanned across the specimen. Low-dose SED was performed with an electron fluence of $\sim 5 \mathrm{e} / \AA^{2}$ and a spatial resolution of $\sim 5 \mathrm{~nm}$ by making use of a counting type Merlin/Medipix direct electron detector. A beam with $\sim 0.6 \mathrm{mrad}$ convergence angle was used to avoid overlapping diffraction disks and with these conditions it was typically possible to obtain a reciprocal space structural resolution of $\sim 1 \AA^{-1}$, as in Figure 1a. The 4D-SED data, thus obtained, contain a wealth of spatially resolved crystallographic data, which may be analyzed in real and reciprocal space. Combined with a wealth of computational analysis, which here includes post-facto imaging and newly developed orientation mapping methods specifically for low-signal data, the technique is a highly versatile hybrid diffraction-imaging microscopy.

Pharmaceutical nanostructure was explored by applying SED microscopy to mechanochemically transformed powders produced using various ball milling conditions applied to pure APIs, paracetamol:co-former mixtures [4], and mixtures in the paracetamol-theophylline-caffeine system. All pure compounds were purchased off-the shelf and the mechanical treatment was intended to induce a range of solid-state processes and reactions, which may be considered mechanochemical. This parallels the increasing use of mechanochemical methods in the pharmaceutical context to screen solid forms of APIs and their co-crystals [5] and is of fundamental interest since many industrial processing routes involve mechanical processes, e.g. milling and blending, which could potentially induce 
mechanochemical transformation. Samples were prepared for SED microscopy by transferring powders directly from the ball mill to standard carbon coated (S)TEM support grids.

Nanostructure was observed in all mechanochemically transformed powders. A particularly interesting example was the observation of paracetamol-theophylline co-crystals (KIGLUI01, $P 2{ }_{1} / n, a=8.751 \AA, b$ $=15.393 \AA, c=11.620 \AA, \beta=99.02^{\circ}$, KIGLUI01) with a rod-like morphology and a twisted structure, as in Figure 1. The average diffraction pattern from the region of interest (Figure 1a) was characteristic of a fiber pattern and virtual dark-field images (Figure 1c), produced by plotting the intensity within a selected disk in the diffraction plane as a function of probe position, gave bands along the rod axis for reflections near to the twist axis and perpendicular to the rod axis for reflections normal to the twist axis. These observations revealed the twisted structure of the rod and showed that the rod and twist axes are aligned. A diffraction pattern from near to the tip of the rod (Figure 1b) could be indexed to the crystal orientation illustrated in Figure 1d. Rotating this crystal structure about an axis perpendicular to the rod/twist axis (Figure 1e) suggests that the twist axis may be perpendicular to hydrogen bonded planes in the crystal structure. This example further establishes low-dose SED as a powerful platform for the investigation of nanostructure in pharmaceutical materials and other organic solids [6].

\section{References:}

[1] MD Eddleston \& W Jones in "Disordered Pharmaceutical Materials", ed. M Descamps, (Wiley-VCH Verlag GmbH \& Co, Weinheim) (2016), pp. 103-134.

[2] DN Johnstone \& PA Midgley, Microsc. Microanal. 23 (2017), p. 1192.

[3] O Panova et al, Micron, 88 (2016,) p. 30.

[4] S Kaki et al, Advanced Materials 21 (2009), p. 3905.

[5] D Hasa \& W Jones, Adv. Drug Deliv. Rev. 117 (2017), p. 147.

[6] The authors acknowledge financial support from the GSK-Cambridge Varsity Alliance. We thank Diamond Light Source Ltd. for access and support in the use of the electron Physical Science Imaging Centre (EM16983, EM17991, EM18488, EM20527).

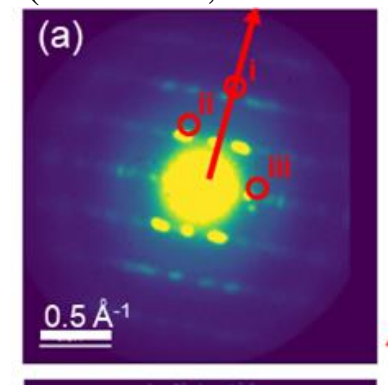

(c)
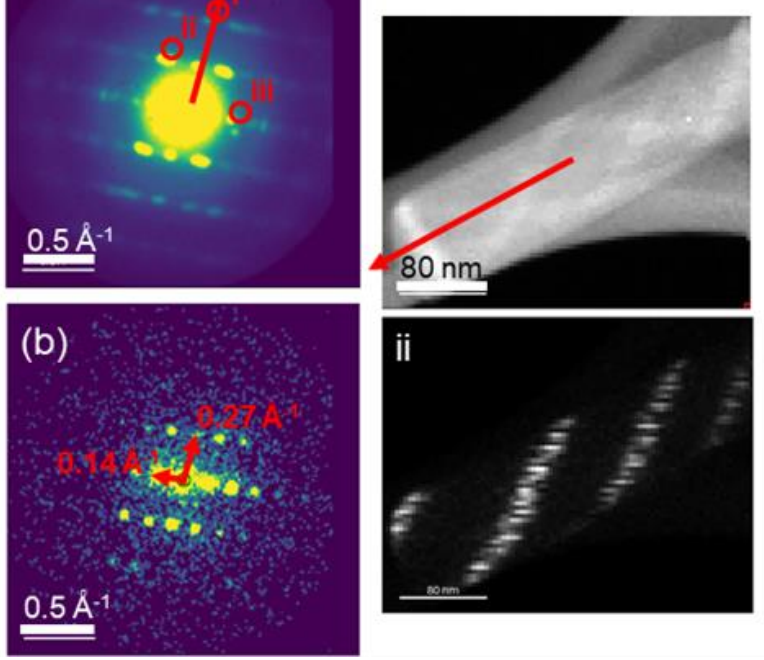

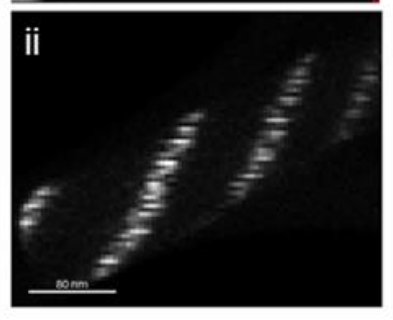

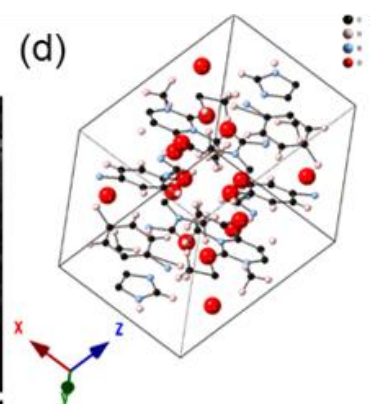
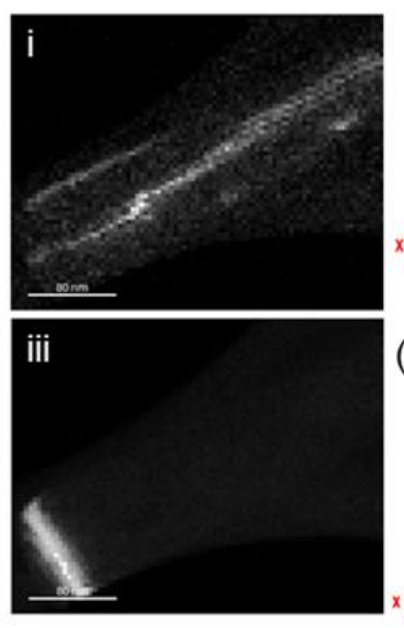

(e)

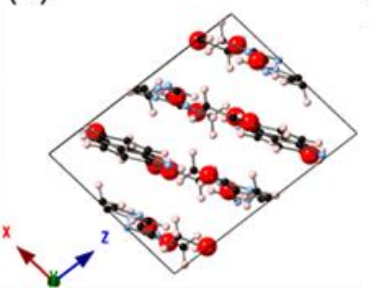

Figure 1. SED microscopy of a paracetamol-theophylline co-crystal. (a) Spatially averaged diffraction pattern, (b) individual diffraction pattern from near the tip of the rod. (c) Dark-field images formed by plotting the intensity within the disks marked in (a) as a function of probe position. (d) Crystal orientation associated with the pattern shown in (b). (e) Rotated structure showing that the twist axis may be perpendicular to hydrogen bonded planes. 